OPEN ACCESS

Edited by:

Paivi H. Torkkeli,

Dalhousie University, Canada

Reviewed by:

Andrew Mason,

University of Toronto Scarborough,

Canada

Andrew Dacks,

West Virginia University, USA

*Correspondence: Alan R. Kay

alan-kay@uiowa.edu

Specialty section:

This article was submitted to Invertebrate Physiology, a section of the journal Frontiers in Physiology

Received: 14 July 2016 Accepted: 26 August 2016 Published: 12 September 2016

Citation:

Kay AR, Raccuglia D, Scholte J, Sivan-Loukianova E, Barwacz CA, Armstrong SR, Guymon CA, Nitabach MN and Eberl DF (2016) Goggatomy: A Method for Opening Small Cuticular Compartments in Arthropods for Physiological Experiments. Front. Physiol. 7:398. doi: 10.3389/fphys.2016.00398

\section{Goggatomy: A Method for Opening Small Cuticular Compartments in Arthropods for Physiological Experiments}

\author{
Alan R. Kay ${ }^{1 *}$, Davide Raccuglia ${ }^{2}$, Jon Scholte ${ }^{3}$, Elena Sivan-Loukianova ${ }^{1}$, \\ Christopher A. Barwacz ${ }^{4}$, Steven R. Armstrong ${ }^{4}$, C. Allan Guymon ${ }^{3}$, Michael N. Nitabach ${ }^{2}$ \\ and Daniel F. Eberl ${ }^{1}$ \\ ${ }^{1}$ Department of Biology, University of lowa, lowa, IA, USA, ${ }^{2}$ Department of Cellular and Molecular Physiology, Yale University, \\ New Haven, CT, USA, ${ }^{3}$ Department of Chemical Engineering, University of lowa, Iowa, IA, USA, ${ }^{4}$ Department of Dentistry, \\ University of lowa, Iowa, IA, USA
}

Most sense organs of arthropods are ensconced in small exoskeletal compartments that hinder direct access to plasma membranes. We have developed a method for exposing live sensory and supporting cells in such structures. The technique uses a viscous light cured resin to embed and support the structure, which is then sliced with a sharp blade. We term the procedure a "goggatomy," from the Khoisan word for a bug, gogga. To demonstrate the utility of the method we show that it can be used to expose the auditory chordotonal organs in the second antennal segment and the olfactory receptor neurons in the third antennal segment of Drosophila melanogaster, preserving the transduction machinery. The procedure can also be used on other small arthropods, like mosquitoes and mites to expose a variety of cells.

Keywords: insects, Drosophila melanogaster, sensory receptor cells, imaging, genetically encoded sensors, mosquitoes, ants, mites

\section{INTRODUCTION}

Most arthropod sense organs are embedded in cuticular compartments and are relatively inaccessible. It is possible to record from bristle organ sensory cells after clipping off the bristle tip and applying an electrode to the cut end (Corfas and Dudai, 1990). Drosophila's Johnston's organ (JO), which constitutes the fly's ear is enclosed in a cuticular chamber, the second antennal segment (A2) and although it has been possible to record extracellularly from its afferent nerve process (Eberl et al., 1997), it has not as yet been possible to record intracellularly from the constituent cells of the chordotonal organ, as it has in larger insects (Hill, 1983; Field and Matheson, 1998).

Although, it is now possible to patch-clamp Drosophila CNS in semi-intact preparations (Wilson et al., 2004), the exoskeleton has for the most part thwarted access to neurons and sensory structures buried within small cuticular compartments. Pioneering work by Dubin and Harris (1997) showed that it was possible to obtain patch clamp recordings from olfactory sensory neurons in Drosophila's third antennal segment (A3), cut open with iridectomy scissors. However, there is a need for a procedure that makes access simpler, more accurate and applicable to even smaller compartments. Providing open access to these compartments is essential if one is to be able to voltage clamp the cells, do single channel recordings or apply pharmacological agents. 
In this communication we describe a method for opening the exoskeleton of arthropods that can be used to access small compartments not amenable to conventional microscopic dissection and expose, in a live state, the enclosed cells. There is a long history of using sliced arthropod eye preparations in neuroscience (Hartline et al., 1952; Hadjilazaro and Baumann, 1968; Wu and Pak, 1975). Our method modifies and extends this approach, by using a viscous photo-polymerizable resin that can be rapidly light cured, to support and anchor the cuticle. We believe that the method could be of value in a variety of physiological and pharmacological experiments on Drosophila's JO and many other arthropod organs, appendages and brains.

\section{MATERIALS AND METHODS}

\section{Drosophila Strains}

For initial tests to develop the protocol, we used a Canton $S$ wild type strain. To visualize membranes of all neurons in the fly, including those in the antenna, we used a $w$ elav ${ }^{C 155}$. Gal4 UAS-mCD8-GFP strain (Bloomington Drosophila Stock Center stock \#5146). The elav ${ }^{C 155}$-Gal4 driver expresses in all neurons (Lin and Goodman, 1994). For $\mathrm{Ca}^{2+}$ imaging, we crossed $w$ elav ${ }^{C 155}$-Gal4 females (Bloomington Drosophila Stock Center stock \#458) with homozygous $w$; UAS-GCaMP6m males (Bloomington Drosophila Stock Center stock \#42748). To record voltage-dependent fluorescence changes, we crossed homozygous $w$; JO15-2-Gal4 females to $w$; UAS-ArcLight ${ }^{\text {attP40 }}$ homozygous males (Bloomington Drosophila Stock Center stock \#51057) (Cao et al., 2013). The JO15-2 line, which expresses in the JO-A and JO-B subgroups of JO neurons, was derived from the original third chromosome JO15 insertion (Sharma et al., 2002; Kamikouchi et al., 2006) by P-element remobilization to the second chromosome.

\section{Drosophila Saline}

Our saline was based on the formulations of Wilson and Laurent (2005) and Hardie et al. (2002). To optimize the recording we used a goggatomized Drosophila eye preparation where extracellular potentials in the retina were recorded with a glass electrode. Oxygenation of the saline proved unnecessary for sustaining the vitality of the preparations.

Drosophila saline (DS) in mM: $120 \mathrm{NaCl}, 3 \mathrm{mM} \mathrm{KCl}, 1 \mathrm{CaCl}_{2}$, $4 \mathrm{MgCl}_{2}, 4 \mathrm{NaHCO} 3,1 \mathrm{NaH}_{2} \mathrm{PO}_{4}, 8 \mathrm{D}$-trehalose, $5 \mathrm{D}$-glucose, 2.5 L-alanine, 2.5 L-proline, 5 L-glutamine, and 5 TES (pH 7.15).

\section{Imaging and Electrophysiology}

Preparations were inserted into $\sim 1 \mathrm{~mm}$ ball of soft dental wax (white square ropes, Heraeus Kulzer, South Bend, IN) melted onto a $12 \mathrm{~mm}$ diameter cover glass that was placed in a perfusion chamber (Siskiyou Corp., Grants Pass, OR) and imaged on an Olympus BX50WI upright microscope equipped with a Hamamatsu ORCA-Flash 4.0 CMOS camera, with illumination provided by an X-Cite 120 LED (Excelitas Technologies Corp., Waltham, MA) through a Semrock (Rochester, NY) BrightLine filter set (472/30 Bandpass, 495 Dichroic and a 520/35 Bandpass) and controlled by MetaMorph software (Molecular Devices, Sunnyvale, CA).
The images in Figure 8 were acquired on a Zeiss Axio Examiner upright microscope using a Plan Apochromat $40 \times$ N.A. 1.0 water immersion objective (Zeiss, Germany), using a Colibri LED system (Zeiss, Germany) with excitation at 470 $\mathrm{nm}$. The objective C-mount image was projected onto the $80 \times 80$ pixel chip of a NeuroCCD-SM camera controlled by NeuroPlex software (RedShirtImaging, Decatur, GA). For image demagnification we used an Optem C-to-C mount 25-70-54 0.38 $\times$ (Qioptiq LINOS, Fairport, NY).

For scanning electron microscopy, goggatomized preparations were fixed overnight in $2.5 \%$ paraformaldehyde and $2 \%$ glutaraldehyde in $0.1 \mathrm{M}$ phosphate buffer $(\mathrm{pH} 7.4)$ at $4^{\circ} \mathrm{C}$, then rinsed with phosphate buffer, dehydrated, subjected to critical point drying, mounted on stubs and coated with gold. The samples were examined with a Hitachi S-4800 scanning electron microscope.

Cells were stimulated with a glass microelectrode ( $\sim 5 \mathrm{M} \Omega)$ filled with DS which was controlled by pClamp software (version 9) through a Digidata 1322A (Molecular Devices, Sunnyvale, CA) coupled to an AMPI Iso-Flex stimulus isolator.

\section{Light Cured Resin (LCR)}

The LCR composition: 70\% BisGMA (bisphenol A diglycidyl methacrylate), 28.75\% HEMA (2-hydroxy methacrylate), $1 \%$ EDMAB (2-ethyl dimethyl-4-aminobenzoate) and $0.25 \%$ CQ (camphorquinone).

LCR was cured with a SDI Radii Plus LED curing light with a light intensity of $1.5 \mathrm{~W} \mathrm{~cm}^{-2}$ and a peak at $460 \mathrm{~nm}$.

A tungsten needle or electrode ( $\sim 28$ gauge and $\sim 2^{\prime \prime}$ long) can be used for picking up a larger bead of LCR that can be used for the conventional goggatomy. For the free-arista-goggatomy smaller quantities of LCR are needed. In this case a tungsten needle can be coated with a thin layer of hard dental wax that the LCR wets (regular stick wax, Whip Mix Corp., Louisville, $\mathrm{KY}$ ). The cured LCR is cut with a carbon steel Feather blade (Ted Pella Inc.).

\section{Analysis of the LCR}

Real Time Fourier Transform Infrared spectroscopy (FTIR) studies were performed using Nicolet Fisher Nexus 670. Samples of the LCR were placed between two sodium chloride plates using $25 \mu \mathrm{m}$ spacer beads. Conversion was evaluated using the absorption band at $1636 \mathrm{~cm}^{-1}$.

Differential Scanning Calorimetry (DSC) studies were performed using a Perkin Elmer Diamond Differential Scanning Calorimeter modified to allow illumination of polymer samples. Heat evolved from the polymerization reaction was used to evaluate reaction behavior using a plain aluminum pan as a reference.

For both Real Time FTIR and DSC reactions were monitored for $3 \mathrm{~min}$ to evaluate reaction duration. All experiments were performed using a Rembrandt Allegro ${ }^{\mathrm{TM}}$ lamp. A light intensity of $1.5 \mathrm{~W} \mathrm{~cm}^{-2}$ with peak irradiance at $450 \mathrm{~nm}$.

All chemicals were from Sigma-Aldrich. All data are expressed as mean \pm SEM, with all the data measured from different preparations. All mean responses, unless otherwise noted, were 
significantly different from the baseline noise as judged by a two-tailed Student $t$-test with $p<0.001$.

\section{RESULTS}

The method described here is simple; the body part is coated with a custom formulated light cured resin (LCR), which is applied as a viscous liquid and then cured to a solid by exposure to light. The sample embedded in the cured compound is then sliced with a fine razor blade while covered with a physiological saline (Figure 1). The cured resin supports and reinforces the exoskeleton as the blade moves through it preventing its collapse

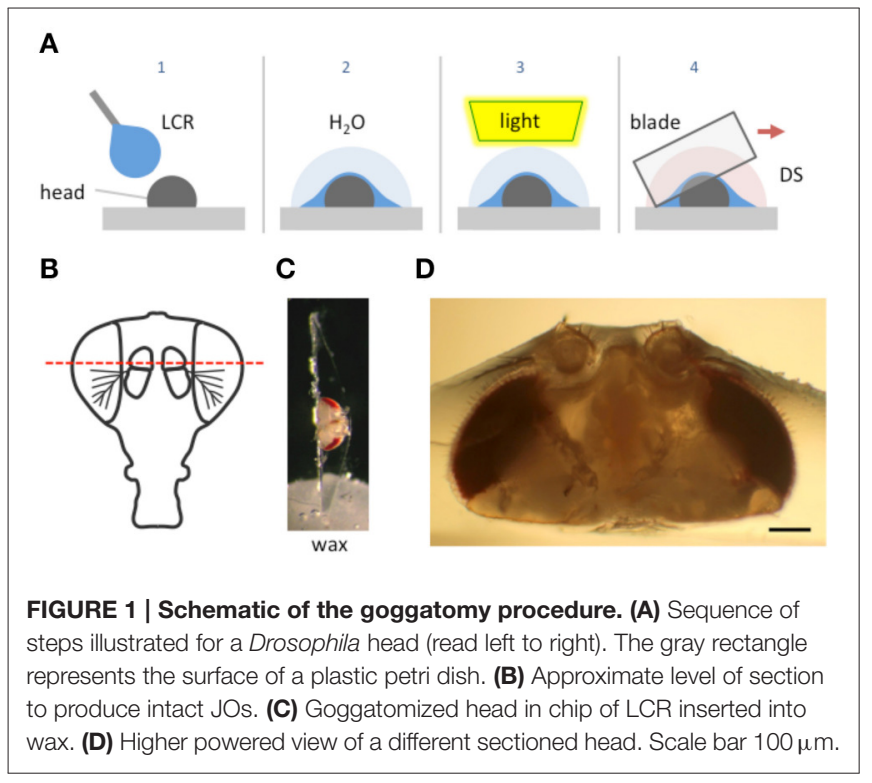

and provides a handle for manipulating and positioning the sectioned material.

We have called the procedure a "goggatomy," from the South African word for insect, gogga (/'xрxə/ http://www. oxforddictionaries.com/definition/learner/gogga) which derives from the Khoisan language; the original inhabitants of South Africa who have a unique click based language (Haacke and Eiseb, 2002).

The LCR used for this procedure is clear and becomes hard after a few seconds of irradiance with a blue light $(460 \mathrm{~nm})$. We will also show how it can be used to affix insects to a substrate and how it can be used to aid the viewing of neurons in intact animals.

We illustrate the method using the A2 of Drosophila melanogaster. The head of a cold-anesthetized fly is cut off with a blade and placed posterior side down on the cover of a 35 $\mathrm{mm}$ plastic petri dish. Details of the procedure are given in the Appendix and an online video is available at http://www.youtube. com/watch? $\mathrm{v}=\mathrm{rH} 5 \mathrm{aOc7ZKjY}$ and in Supplementary Material. A small drop of LCR is applied to the anterior side of the head and allowed to flow over it. The droplet should be a little larger than the head $(\sim 2 \mu \mathrm{l})$ and is applied with a thin needle. Once the resin has covered the head a small drop of water $(\sim 10 \mu \mathrm{l})$ is placed over the LCR. The resin is then cured with a dental curing light held within a few millimeters of the LCR drop for $1 \mathrm{~min}$. The water serves two purposes: (1) It reduces the amount of oxygen, which inhibits curing of the outer layer of resin. (2) It helps dissipate heat (vide infra). The water is wiped off the cured drop with a tissue and a drop of Drosophila saline (DS-see Methods) is placed over the cured LCR. A new blade is cleaned with ethanol, broken into quarters and used to slice through the embedded head. Under a dissecting microscope, the blade is carefully oriented in the plane that one desires to cut. The blade is then firmly and quickly drawn through the encapsulated head, cleaving it into two pieces. As soon as the cuticle is breached,

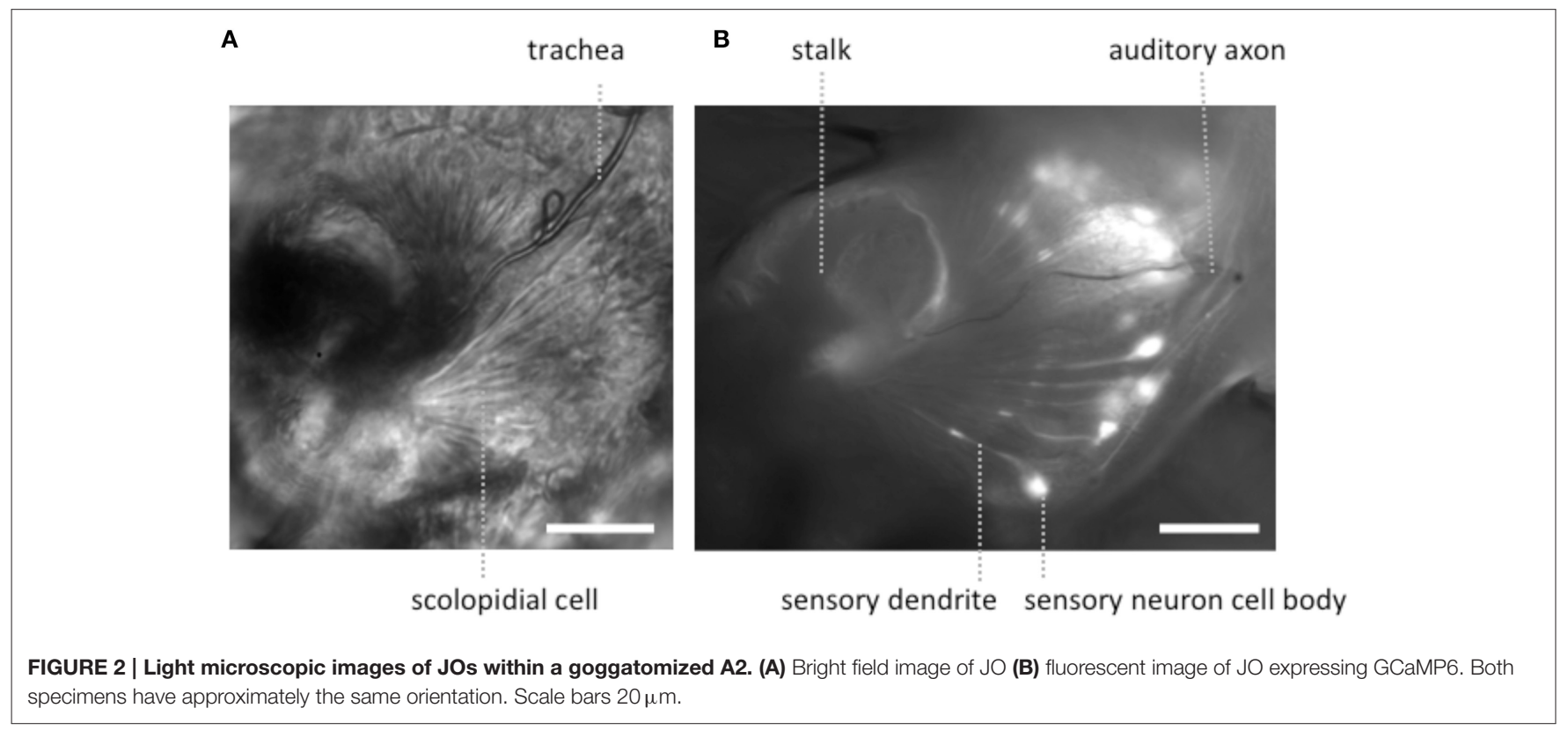


DS flows into the cut. The cuticle is securely embedded in the cured LCR and the separated LCR chips can be handled with forceps and placed in a dish with DS. The cured LCR is denser than water and sinks the sample to the bottom of the dish. To hold and position the sample we use a small piece of soft dental wax melted onto a $12 \mathrm{~mm}$ circular coverslip. An edge of the LCR chip containing the sample is simply pressed into the wax at the appropriate orientation.

We use the top of a $35 \mathrm{~mm}$ tissue culture dish as a work surface, since it forms an ideal platform that can be held and orientated with one hand while the other cuts through the embedded insect. Moreover, the LCR bonds tightly to the surface of the dish so that the preparation remains fixed as the blade is drawn through it.

\section{LCR Composition and Properties}

LCRs are widely used in dentistry to create permanent durable implants. The chemical components from which they are formulated have a long history of use in the human oral cavity and extensive tests have established their safety (Pereira et al., 2009). We screened five published formulations (Pashley et al., 2007). Of these, their resin \#3 (see Methods and Materials for composition) proved best in terms of its ability to support the exoskeleton, retain the tissue after cutting and bond to the dish.
The latter is important since the LCR should adhere to the dish as the blade moves through the sample. The cured resin fractures along the line of the cut rather than shattering. Moreover, this formulation is optically clear with a refractive index of 1.57 that facilitates index-matching improving optical resolution. It is worth noting that light cured adhesives have been used to mount Drosophila for in vivo recordings (Budick et al., 2007; Seelig et al., 2010).

Polymerization of the LCR was followed by real time FTIR. The average conversion of the resin was $68 \%(n=3)$ after $90 \mathrm{~s}$ of illumination. At this point the polymer matrix has vitrified so that the diffusion of residual monomer would be slow. Similar experiments performed with DSC found that most of the reaction occurred within the first $20 \mathrm{~s}$ of illumination.

When the LCR polymerizes, its density increases and hence it shrinks. Under the conditions employed here, where an unconstrained thin layer is applied to the insect the shrinkage is likely to be non-uniform. The fact that it is cured under water might have an influence on this too. The surface of the cured LCR appears reticulated, which further supports a non-uniform curing process. Moreover, after cutting through the A2, its profile does not appear to be deformed, as might occur if the shrinkage of the LCR squeezed the antenna appreciably.

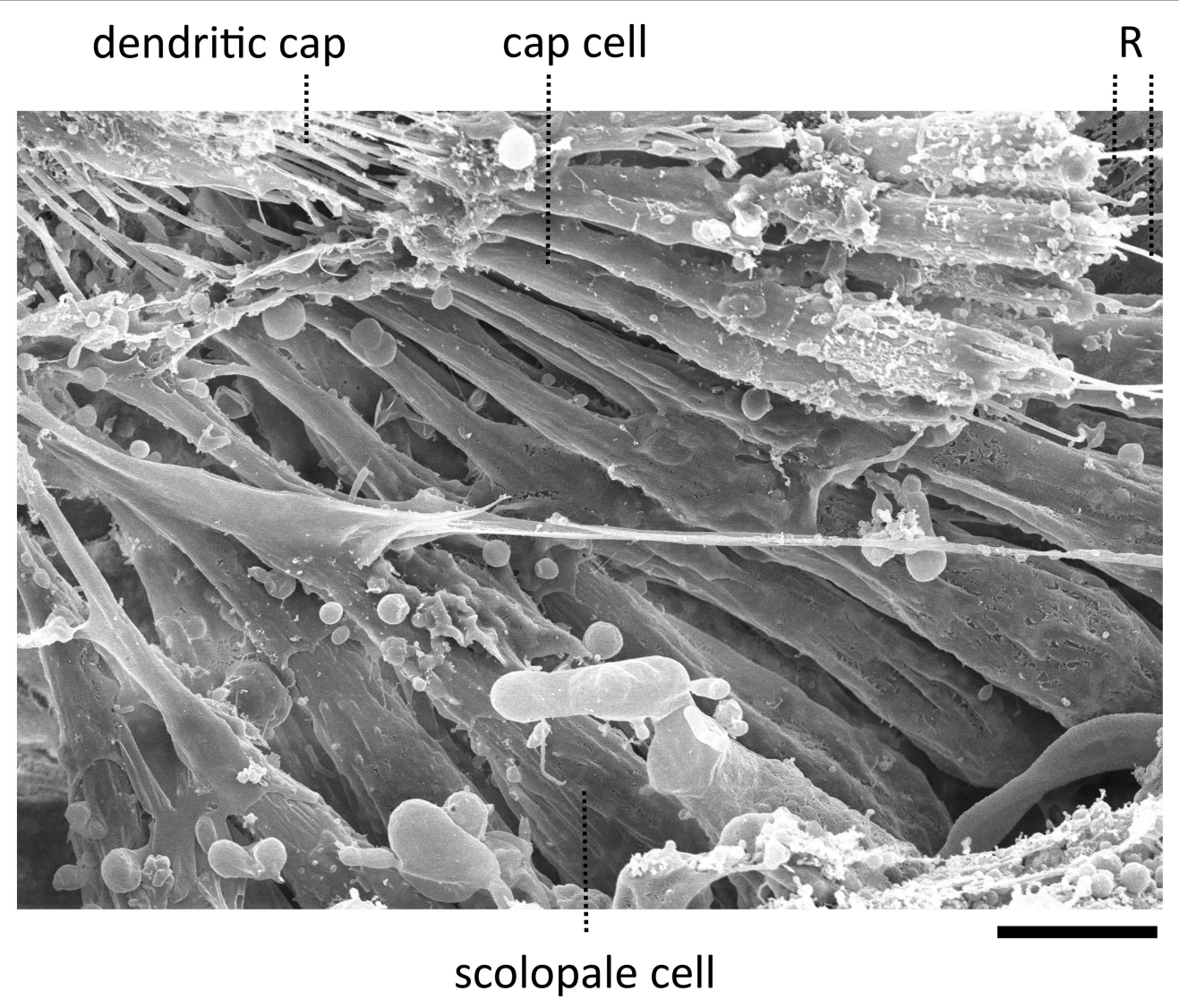

FIGURE 3 | Scanning electron micrograph of a JO within a goggatomized A2. R-putative scolopale rods protruding from broken scolopidia. Scale bar 5 um. 


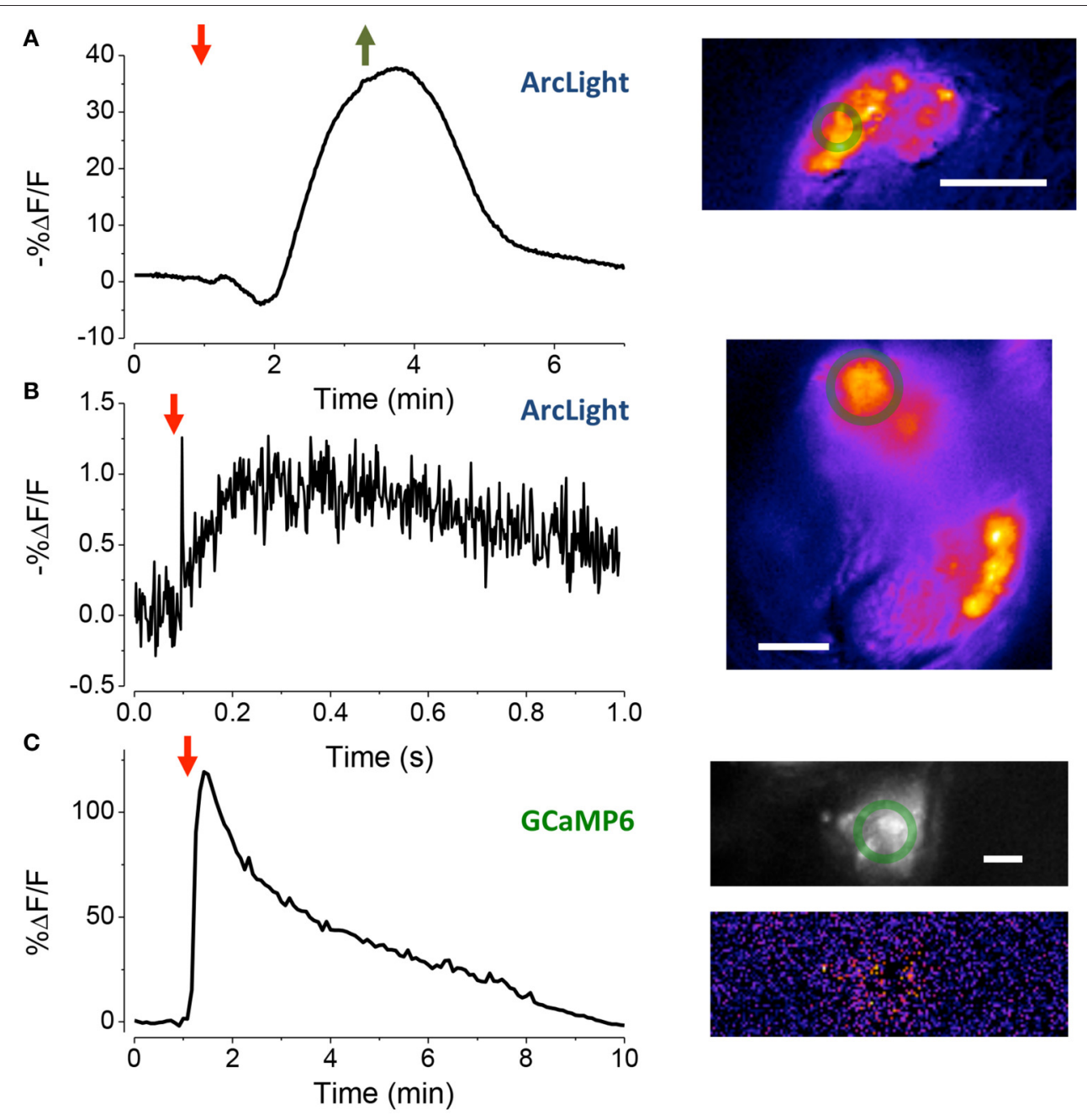

FIGURE 4 | Assessing the vitality of exposed JOs. (A) Response of sensory cells in JO expressing ArcLight to the perfusion of HiK (red arrow) and restoration of normal saline (green arrow). Inset, pseudo-color image of difference between the image prior to stimulation and at its peak. Scale bar $20 \mu \mathrm{m}$. The green circles are the regions-of-interest used to produce the time traces. (B) Local electrical stimulation (arrow, $200 \mu \mathrm{s}, 0.1 \mathrm{~mA}$ ) of sensory cells in JO expressing ArcLight. Scale bar 10 $\mu \mathrm{m}$. (C) Response of JO expressing GCaMP6 to application of pymetrozine (arrow, 3.4 $\mu \mathrm{M}$ ). Top inset fluorescence, bottom inset pseudo-color difference image. Scale bar $20 \mu \mathrm{m}$.

Two important aspects of the LCR are its viscosity and its ability to wet arthropod cuticles. The high viscosity of the LCR $(\sim 1200 \mathrm{cP})$ allows one to suspend a droplet about the size of a fly's head without it dropping off the applicator. The viscosity of the LCR slows its spread along the cuticle, which allows one to cover only part of the head or body, if so desired (vide infra). The wetting characteristics of the LCR induce it to penetrate between the setae and into even the finest crevices in the insect cuticle. This ensures that LCR holds the fly part, as the resin does not actually bond to the insect cuticle.

The LCR formulation used here does not generate much heat during the curing process. We tested the heat generated during curing by applying a drop of LCR to a piece of aluminum foil overlying a thermistor probe. Illuminating the LCR for $1 \mathrm{~min}$ led to an increase in temperature of only $2.6 \pm 0.5^{\circ} \mathrm{C}(n=4$, starting temperature $24.3 \pm 0.7^{\circ} \mathrm{C}$ ). For most physiological applications this is a negligible increase.

\section{Morphology of Sensory Cells Post-goggatomy}

To expose JOs, a fly head embedded in LCR was cut horizontally dorsal to the A2-A3 joint (Figure 1B). At this level of section it was possible to observe intact scolopidia in the dorsal parts of both divided A2s. The form of the JO could be clearly resolved under bright field optics in goggatomized preparations, with the scolopidia attached to the remnants of the stalk (Figure 2). The outer surface of the scolopale cells was bright and lustrous, suggesting that the scolopidia are preserved. Many scolopidia remain attached to the joint cuticle via their dendritic caps. Pushing against the scolopidium at right angles to its long axis with a patch electrode allows one to assess the integrity of this link. If the link is broken the scolopidium swings free.

The goggatomy procedure allows preparations to be made that can be fixed, dehydrated, gold sputtered and viewed on a scanning electron microscope (Figure 3). SEM images show that 

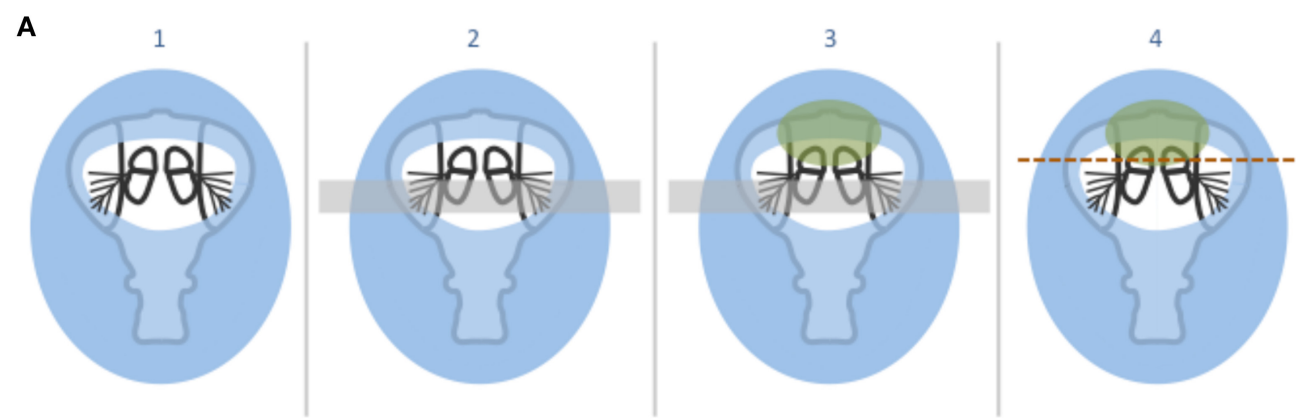

B

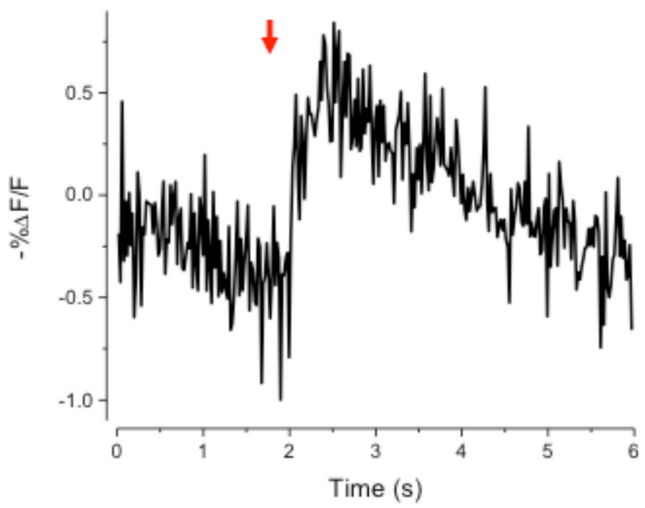

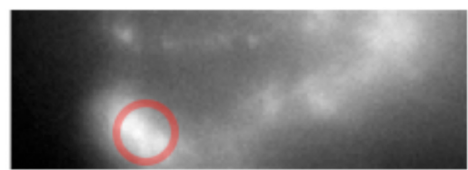

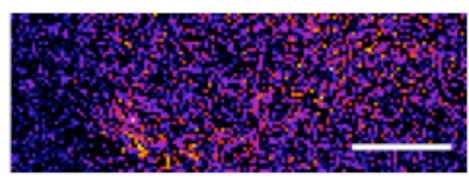

FIGURE 5 | Free-Arista Goggatomy. (A) Schematic of the procedure (1) Place the cut head on a small droplet of LCR (blue) and allowed it to sink so that its edges, but not the antennae or arista, are covered. Cure for $20 \mathrm{~s}$. (2) Place a small strip of colored Cellophane (gray rectangle) over the A3, to prevent LCR from pulling the antennae up when it is applied to A2. (3) Apply a very small droplet of LCR so that it covers the dorsal margins of the A2 (green). Begin light curing, then remove the plastic strip and apply a droplet of water to the preparation. Cure for $1 \mathrm{~min}$. Wick off the water with a tissue. (4) Apply DS and cut through the head as indicated by the dotted line. (B) Response of JO cells expressing GCaMP6 in a FA-goggatomized preparation to an air pulse (120 ms, 1 psi) directed at the solution (red arrow). Inset top, fluorescence, bottom pseudo-color difference image. Scale bar $20 \mu \mathrm{m}$.

much of the scolopidial structure is preserved during goggatomy, even the dendritic caps connecting the scolopale cells to the stalk. The scolopidial cells are plump suggesting that the space is still filled. In some cases where the scolopidia have been cut, dendrites can be seen protruding.

The appearance of the scolopidia under SEM was not as good as that under bright field. We suspect that cells become fragile after dehydration and may fragment. Under saline in bright field microscopy, the full fan-like arrays of scolopidia can be seen (Figure 2), whereas, under SEM this was less common.

\section{Physiology}

In many cases when the head capsule is cut in the horizontal plane, the brain appears to pulse at a rate of $\sim 1 \mathrm{~Hz}$. This is due to the contraction of muscle 16 , the frontal pulsatile organ (Demerec, 1950; Murthy and Turner, 2010), whose pulsations can persist in DS for up to $5 \mathrm{~h}$ in vitro. Moreover, we have used fly lines that express GFP in neurons and in most cases the expression persists after many hours, again suggesting that the exposed cells are viable.

We have also assessed the toxicity of the procedure on whole flies. The dorsal surface of whole flies was attached to a plastic dish with LCR and the survival of the flies was monitored. Flies survived for more than $6 \mathrm{~h}$ and remained fully motile to the extent that the tethering would allow. This survival resembles that when flies are held in pipette tips for extracellular electrophysiology (Eberl et al., 2000). This suggests that if any resin crosses the cuticle it has little toxic effect on the organism.

To assess the electrophysiological vitality of cells in the goggatomized preparations we used the fluorescent voltage sensor ArcLight developed by Pieribone and colleagues (Jin et al., 2012). ArcLight is maximally fluorescent at hyperpolarized potentials, its fluorescence declining roughly linearly as the cell depolarizes. Moreover, its temporal response is sufficiently rapid to follow action potentials with reasonable fidelity. To determine if the sensory cells of JO had a hyperpolarized resting potential, characteristic of live cells, goggatomized A2s were perfused with a saline where all the $\mathrm{Na}^{+}$was substituted by $\mathrm{K}^{+}(\mathrm{HiK})$, which should depolarize the cells to $\sim 0 \mathrm{mV}$. Application of the saline led to a rapid decrease in fluorescence, consistent with depolarization (mean $\% \Delta \mathrm{F} / \mathrm{F}=-28.5 \pm 3.2, n=16$ ) (Figure 4A). If the cells had no resting potential, application of HiK should lead to no or little change in the fluorescence of ArcLight. HiK stimulation induced similar changes in mushroom body neurons expressing ArcLight (mean $\% \Delta \mathrm{F} / \mathrm{F}=-30.7 \pm 5.1$, $n=12$ ). Subjecting sensory neurons expressing GFP to HiK led to no significant change in fluorescence (mean $\% \Delta \mathrm{F} / \mathrm{F}$ $-0.02 \pm 1.4, n=9$ ), indicating that the changes in the 
ArcLight flies are not the result of the cells shrinking or swelling.

To activate sensory neurons directly, a glass microelectrode was placed close to the cell bodies in goggatomized A2s. When a pulse of current was delivered to the electrode, the fluorescence of the cell body declined and then increased back to baseline, consistent with depolarization and then repolarization of the cell (Figure 4B, mean \% $\Delta \mathrm{F} / \mathrm{F}=-1.5 \pm 0.1, n=7$ ).

We also used the insecticide pymetrozine to determine if the scolopidia survive the goggatomy procedure with their transduction mechanism intact. Pymetrozine interacts with the TRPV ion channel complex (with subunits Nan and Iav) resulting in a large influx of calcium (Nesterov et al., 2015). Application of $15 \mu \mathrm{M}$ of pymetrozine to goggatomized A2s from flies expressing GCaMP6 resulted in the consistent and rapid elevation of calcium in the sensory cells (Figure 4C mean \% $\Delta \mathrm{F} / \mathrm{F}=56.3 \pm$ $3.2 \% \Delta \mathrm{F} / \mathrm{F}, n=17)$. Consistent with the reported pymetrozine effect, the response in our preparation was irreversible and could not be restored after washing off the pymetrozine. The sensory neurons do not respond to a second application of pymetrozine even after washing off the drug for $10 \mathrm{~min}$. The decline of fluorescence is not the result of photobleaching, since little occurs with a slow sampling rate $(0.2 \mathrm{~Hz})$ and short exposures $(50 \mathrm{~ms})$.

We have not systematically assessed how long goggatomized preparations can be sustained in a physiologically responsive state in vitro. Our experiments were typically performed within 2 $\mathrm{h}$ after the goggatomy, but recordings of physiological responses were observed even in preparations held for as long as $3 \mathrm{~h}$ in DS.

\section{Free-Arista Goggatomy}

We modified the goggatomy procedure so as to expose the JO while preserving motion about the $\mathrm{A} 2-\mathrm{A} 3$ joint. The procedure is detailed in Figure 5A and is termed the Free-Arista (FA) goggatomy. Directing a small short air pressure pulse toward the surface of the saline moved the arista. Movement of the arista was detected by making a high-speed video and activation of the JO by increases in calcium detected by GCaMP6 (Figure 5B).

\section{Olfactory Receptors}

We further modified the goggatomy procedure to section the A3 while preserving the olfactory receptor neurons (ORNs) (Figure 6B). The approach is detailed in Figure 6A and shows how one can cut the antenna without embedding the sensory sensilla. Bath application of isoamyl-acetate led to a robust response in some of the sensory neurons, showing that their responsiveness is preserved (Figure 6C).

\section{Other Preparations}

The goggatomy procedure is versatile enough that it can also be useful for a number of other exoskeletal compartments besides the antennae. As examples we show sensory cells in the labellum (Figure 7A) and chordotonal organs in the legs (Figures 7B,C). Goggatomy also provides a convenient way of accessing the musculature and cells of the head, proboscis, halteres and sex organs.

In addition, we have found that the goggatomy procedure is applicable to other small arthropods and have tried them

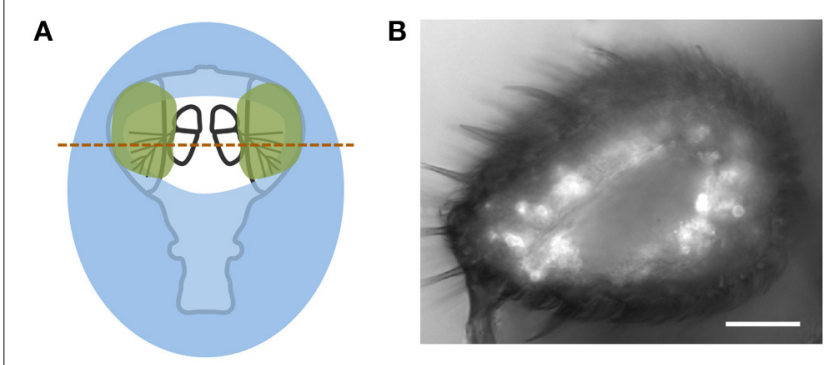

C

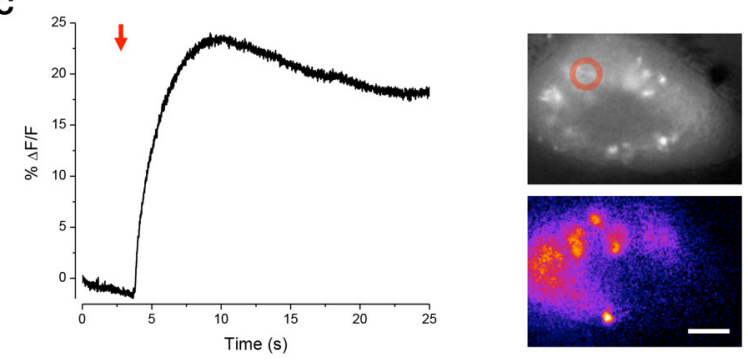

FIGURE 6 | Sectioning A3 and response of ORNs to odorant. (A) Schematic of the procedure. Place the cut head on a droplet of LCR (blue) and allow sinking so that the margins of the head, but not the arista, become covered. Light cure for $20 \mathrm{~s}$. Apply small droplets of LCR over the arista and margins of the antennae (green). Note, the frontal surface of A3 should not be covered so that the sensory sensilla are free. Add droplet of water over preparation and cure for $1 \mathrm{~min}$. Wick off water with tissue. Add droplet of DS and cut through the A3s (red line). (B) Image of goggatomize A3 expressing GCaMP6. (C) Increase in intracellular calcium in ORNs expressing GCaMP6 to the application of iso-amyl acetate (arrow, a $5 \mu \mathrm{l}$ drop of a $0.67 \mathrm{mM}$ solution was added to the $\sim 2 \mathrm{ml}$ bath). Upper inset, fluorescence prior to odorant application. Lower inset pseudo-color difference image. Scale bar $20 \mu \mathrm{m}$.

on the following: mosquitoes (Figure 7D), mites (in Figure 7E), Daphnia, ants and wasps (data not shown).

\section{Using the LCR to View Neurons in Intact Insects}

We have found that we can use the LCR to mount whole live flies under a coverslip to view the antenna with a water immersion objective. The refractive index of cured LCR is 1.57; this makes it possible to preserve the high numerical aperture of the water immersion objectives. The procedure, which we term a "gogga cap," is illustrated in (Figure 8). To image fluorescently labeled scolopidia in A2, while not immobilizing the A2-A3 joint, the following procedure can be followed. A cold-anesthetized fly is affixed to a small piece of fishing line $(0.25 \mathrm{~mm}$ diameter, $\sim 4 \mathrm{~mm}$ length) that is held vertical on a small piece of poster tack under a dissection microscope. To do this, a drop of LCR is placed on the top of the fishing line and the ventral surface of the thorax is placed on the drop of resin. The fly is secured to the line by curing the LCR. A small drop of LCR is applied to a coverslip, which is attached to a coarse micromanipulator via a small rod. Under a stereomicroscope the cover slip is maneuvered over the head and lowered until the resin starts flowing over the head. When the desired coverage is achieved the resin is cured.

In this configuration it is possible to image JO through the cuticle while stimulating the antenna with near field sound and 
A

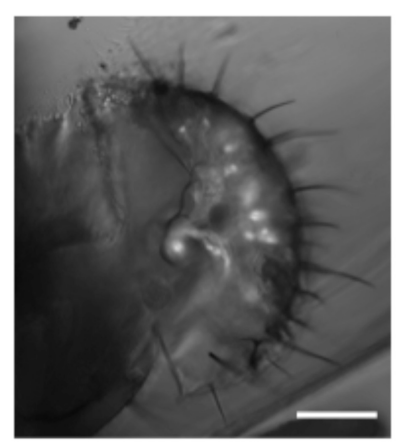

D

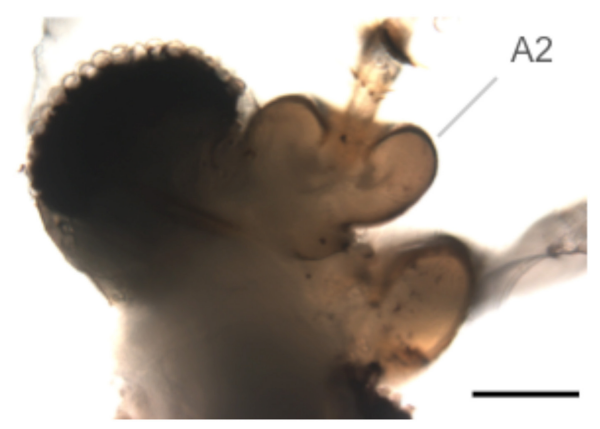

B

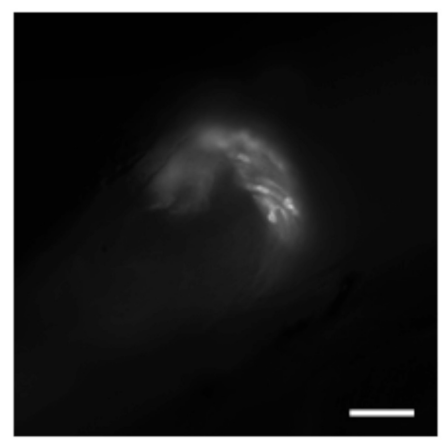

E

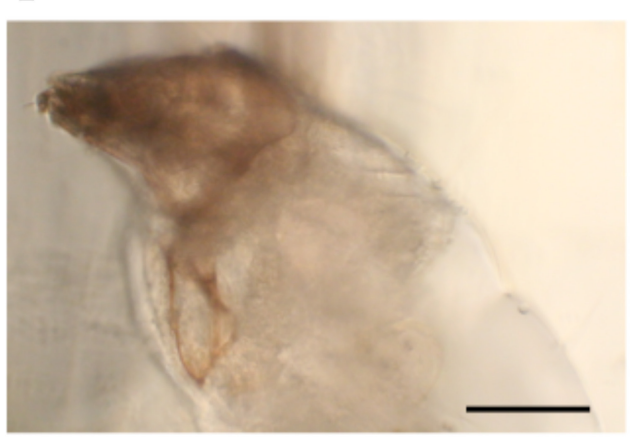

C

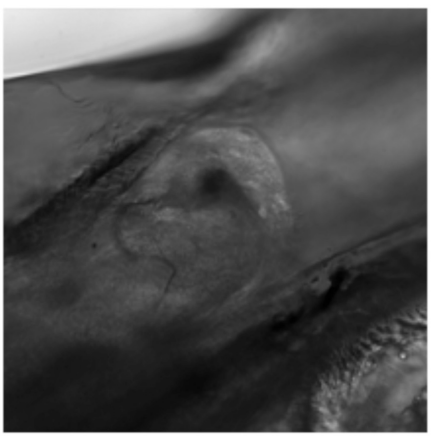

and

FIGURE 7 | Goggatomy of Drosophila and other arthropods. (A) Drosophila labellum, GFP, scale bar 50 $\boldsymbol{\mu m}$. (B,C) Drosophila leg chordotonal organ, GFP, scale bar $25 \mu \mathrm{m}$. (B) Fluorescence and (C) bright field. (D) Mosquito head, Culex pipiens, scale bar $50 \mu \mathrm{m}$. (E) Anterior end of Mimolette cheese mite, Acarus siro, scale bar $50 \mu \mathrm{m}$. Head is top left. The mite was cut in spider saline (Schmitz et al., 2012).

detect changes in potential with ArcLight. An example of such a recording is shown in (Figure 8).

If one wants to image the brain in an intact fly it can simply be attached to a cover slip by holding it head down into a small droplet of LCR on a coverslip while light curing for $1 \mathrm{~min}$.

\section{DISCUSSION}

We have developed a simple procedure for opening up the exoskeleton of arthropods, which exposes the live tissue and neuronal components. The goggatomy procedure opens up previously inaccessible cells for direct physiological and pharmacological manipulation.

We have used the fluorescent voltage sensor ArcLight to show that sensory neurons in JO have a substantial negative potential and auditory sensory neurons respond to current injection. The sensory transduction apparatus of the both JO and ORNs remains intact as judged by chemical stimulation. Moreover, in a preparation where the A2-A3 joint is preserved, sensory neurons respond to joint displacement. In addition, we have found that in Drosophila brain exposed by goggatomy, spontaneous and rhythmic activity persists in vitro (data not shown) (Rosay et al., 2001).

The method does not rely on the LCR adhering to the cuticle. Arthropod cuticles have a thin wax layer and the resin in the uncured form wets it, but when cured does not bond

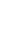

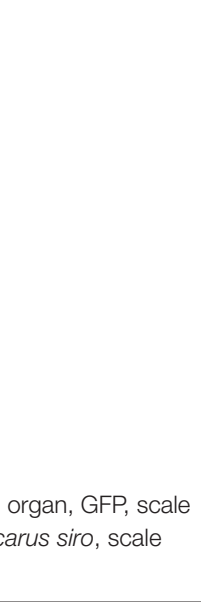



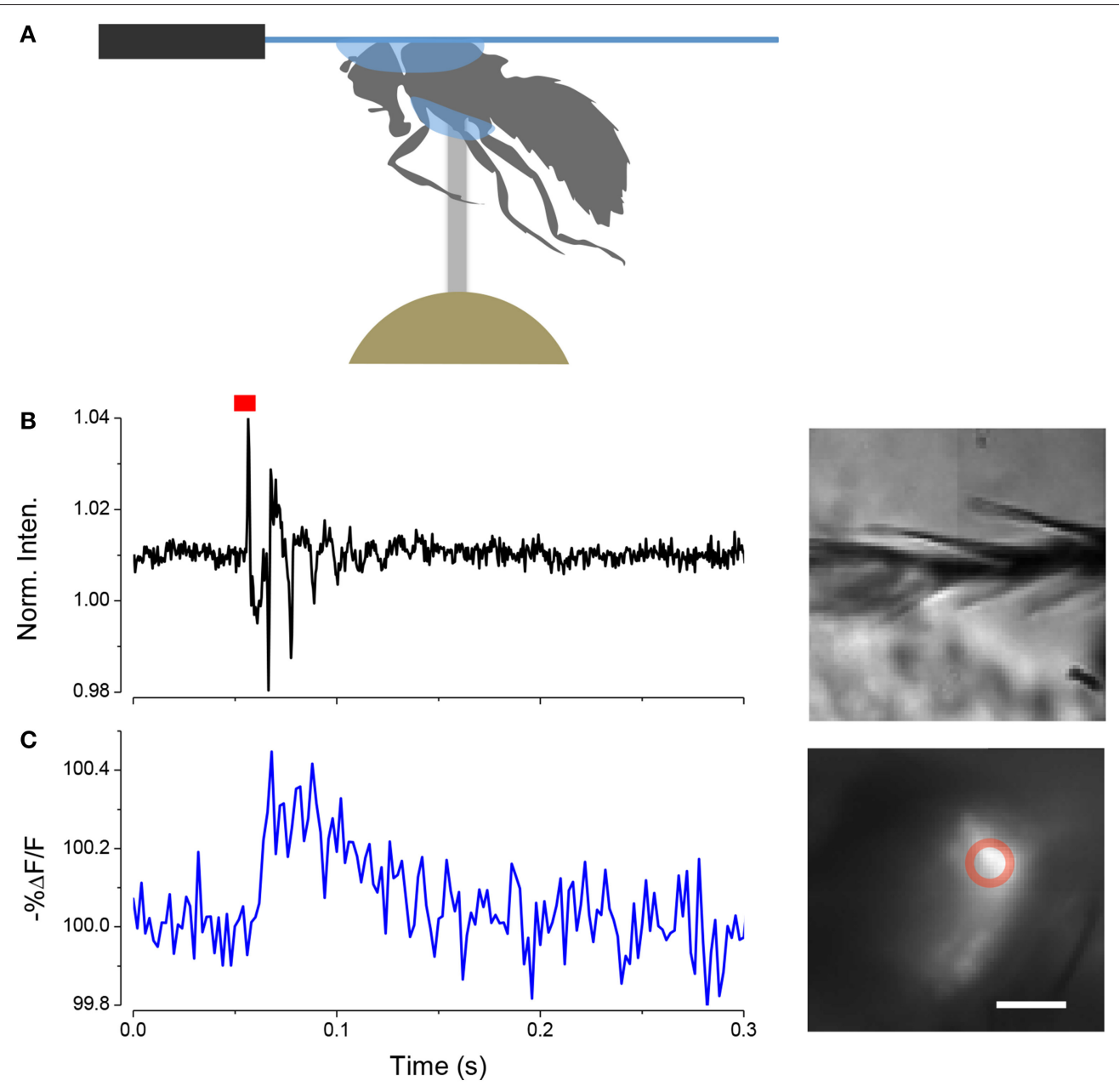

FIGURE 8 | Mounting live flies with LCR. (A) Schematic of the mounting procedure. (B) Movement of arista (inset right) induced by a single sound pulse (red). (C) Response of $\mathrm{JO}$ expressing ArcLight in the same fly as (B). Scale bar $20 \mu \mathrm{m}$.

Sinha et al. (2013) have published a method for cutting holes in insect cuticles with a UV excimer laser, which they used to expose the brain in live Drosophila. They have not used the method to expose cells in small cuticular compartments like the antennae. Our goggatomy procedure is considerably more cost effective and easier to implement. It is worth mentioning that Grover et al. (2016) have developed a method for installing a transparent window in the fly head, which allows the activity of neurons expressing fluorescent probes to be imaged in untethered walking flies.

For arthropods where it is not feasible to use genetically encoded indicators, the goggatomy procedure makes it possible to use the acetoxylmethyl ester (AM) loading of synthetic ionindicators like fluo-4 into cells (Grienberger and Konnerth, 2012) as has been done in the honeybee (Galizia et al., 1999). Drosophila neurons can load with calcein-AM (Li and Meinertzhagen, 1995), however some synthetic calcium probes may not load very efficiently (Jing Wang, personal communication).

Goggatomization of Drosophila heads exposes the beating frontal pulsatile organ (muscle 16) that can be sustained in vitro for a few hours. We suggest that this preparation could be a useful one for studying the rhythmicity of muscle. Moreover, the preparation is simple enough to be used in classroom demonstrations and student labs.

More than 10 years ago Wilson et al. (2004) showed how it was possible to perform patch clamp recordings on adult Drosophila brains. Their method has been widely used opening up this important model organism to physiological investigation. Alan Kay spent 2 years trying to get intracellular recordings from scolopidia without success. The sensory neuron cell bodies are covered by a tough extracellular matrix, which hinders both patch and sharp recordings. The scolopale cells are more rigid than other cells, thwarting attempts to patch and penetrate the cells. Although, we were unable to make direct intracellular recordings from scolopidia, we believe that it would be imprudent to suggest that it is impossible. It might be possible to make intracellular recording from the component cells of scolopidia with an appropriate sharp electrode, perhaps quartz or by digesting the extracellular matrix with proteases. Our confidence that intracellular recordings can be made from Drosophila 

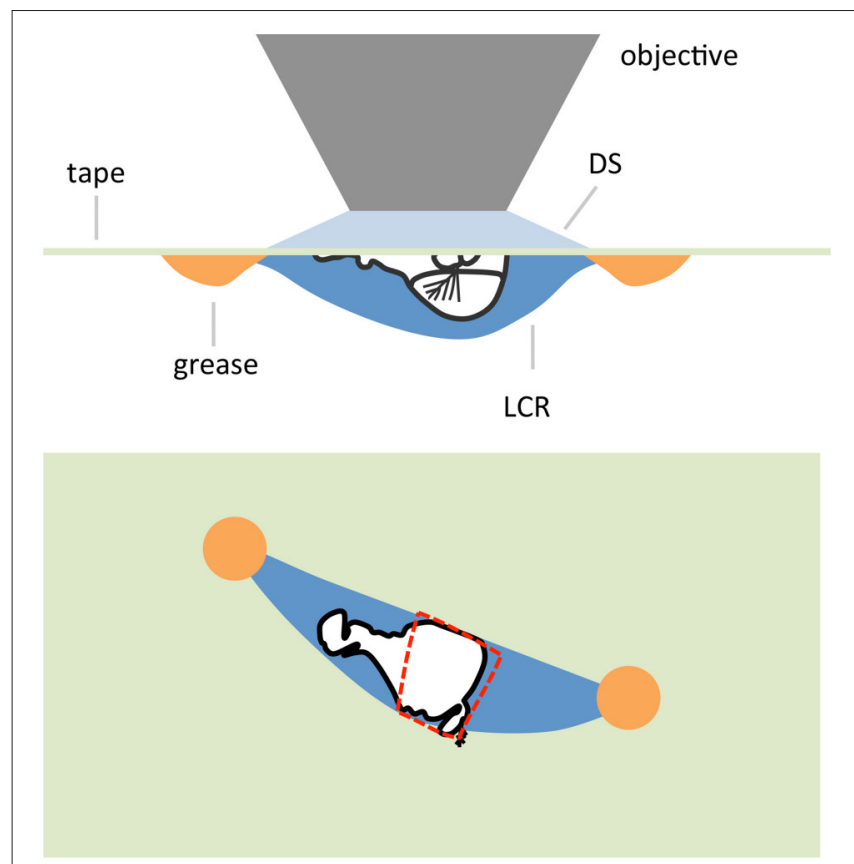

FIGURE 9 | Schematic of an air-gogga preparation. Upper panel, side view. Lower panel, top down view. The dashed red line represents a hole cut into the tape to allow saline to enter the preparation.

JO is bolstered by reports of whole-cell and perforated patch recordings from ORNs in A3s that have been cut open (Dubin and Harris, 1997; Cao et al., 2016).

Isolated neuronal preparations have played an important role in the progress of neuroscience. These include among others, the squid giant axon and synapse, the limulus eye, and isolated

\section{REFERENCES}

Budick, S. A., Reiser, M. B., and Dickinson, M. H. (2007). The role of visual and mechanosensory cues in structuring forward flight in Drosophila melanogaster. J. Exp. Biol. 210, 4092-4103. doi: 10.1242/jeb.006502

Cao, G., Platisa, J., Pieribone, V. A., Raccuglia, D., Kunst, M., and Nitabach, M. N. (2013). Genetically targeted optical electrophysiology in intact neural circuits. Cell 154, 904-913. doi: 10.1016/j.cell.2013.07.027

Cao, L. H., Jing, B. Y., Yang, D., Zeng, X., Shen, Y., Tu, Y., et al. (2016). Distinct signaling of Drosophila chemoreceptors in olfactory sensory neurons. Proc. Natl. Acad. Sci. U.S.A. 113, E902-E911. doi: 10.1073/pnas.1518329113

Corfas, G., and Dudai, Y. (1990). Adaptation and fatigue of a mechanosensory neuron in wild-type Drosophila and in memory mutants. J. Neurosci. 10, 491-499.

Demerec, M. (1950). Biology of Drosophila. New York, NY: Hafner Publishing Co. Inc.

Denno, M. E., Privman, E., Borman, R. P., Wolin, D. C., and Venton, B. J. (2016). Quantification of histamine and carcinine in Drosophila melanogaster tissues. ACS Chem. Neurosci. 7, 407-414. doi: 10.1021/acschemneuro.5b00326

Dubin, A. E., and Harris, G. L. (1997). Voltage-activated and odor-modulated conductances in olfactory neurons of Drosophila melanogaster. J. Neurobiol. 32, 123-137.

Eberl, D. F., Duyk, G. M., and Perrimon, N. (1997). A genetic screen for mutations that disrupt an auditory response in Drosophila melanogaster. Proc. Natl. Acad. Sci. U.S.A. 94, 14837-14842. doi: 10.1073/pnas.94.26.14837 gastropod ganglia. We believe that the goggatomy procedure will be of great value in helping to reveal the secrets of sensory organs and other cells trapped within the confines of very small cuticular compartments.

\section{AUTHOR CONTRIBUTIONS}

$\mathrm{AK}$ and DE initiated the project. AK performed the experiments. $\mathrm{AK}, \mathrm{DR}$, and DE analyzed the data. DE, DR, and MN produced the fly lines. ES performed the SEM. JS, CG and SA made and analyzed the LCR. MN, CB, JS, CG, and SA provided reagents. $A K$ wrote the paper with contributions from all other authors.

\section{ACKNOWLEDGMENTS}

We thank Mark Reagan and Matthew Wortel for measuring the refractive index of the LCR, Lyric Bartholomay for mosquitoes, Andrew Forbes for wasps and the New Pioneer Coop for the mimolette cheese, Baruch Minke for helpful advice, and Roger Hardie, Toshihiro Kitamoto and Jing Wang for comments on an earlier version of the manuscript. The work was in part supported by an award from the Office of the Vice President for Research, University of Iowa (AK) and by the Iowa Center for Molecular Auditory Neuroscience, supported by NIH P30 grant DC010362 to Steven Green.

\section{SUPPLEMENTARY MATERIAL}

The Supplementary Material for this article can be found online at: http://journal.frontiersin.org/article/10.3389/fphys. 2016.00398
Eberl, D. F., Hardy, R. W., and Kernan, M. J. (2000). Genetically similar transduction mechanisms for touch and hearing in Drosophila. J. Neurosci. 20, 5981-5988.

Field, L. H., and Matheson, T. (1998). Chordotonal organs of insects. Adv. Insect Physiol. 27, 1-230. doi: 10.1016/S0065-2806(08)60013-2

Galizia, C. G., Sachse, S., Rappert, A., and Menzel, R. (1999). The glomerular code for odor representation is species specific in the honeybee Apis mellifera. Nat. Neurosci. 2, 473-478. doi: 10.1038/8144

Grienberger, C., and Konnerth, A. (2012). Imaging calcium in neurons. Neuron 73, 862-885. doi: 10.1016/j.neuron.2012.02.011

Grover, D., Katsuki, T., and Greenspan, R. J. (2016). Flyception: imaging brain activity in freely walking fruit flies. Nat. Methods 13, 569-572. doi: 10.1038/nmeth.3866

Haacke, W. H. G., and Eiseb, E. (2002). A Khoekhoegowab Dictionary. Windhoek: Gamsberg MacMillan Pub.

Hadjilazaro, B., and Baumann, F. (1968). Afterpotentials of the visual cell of the honey-bee drone. Helv. Physiol. Pharmacol. Acta 26, CR351-CR352.

Hardie, R. C., Martin, F., Cochrane, G. W., Juusola, M., Georgiev, P., and Raghu, P. (2002). Molecular basis of amplification in Drosophila phototransduction: roles for G protein, phospholipase C, and diacylglycerol kinase. Neuron 36, 689-701. doi: 10.1016/S0896-6273(02)01048-6

Hartline, H. K., Wagner, H. G., and Macnichol, E. F. Jr. (1952). The peripheral origin of nervous activity in the visual system. Cold Spring Harb. Symp. Quant. Biol. 17, 125-141. doi: 10.1101/SQB.1952.017. 01.013 
Hill, K. G. (1983). The physiology of locust auditory receptors I. Discrete depolarizations of receptor cells. J. Comp. Physiol. 152, 475-482. doi: $10.1007 / \mathrm{BF} 00606437$

Jin, L., Han, Z., Platisa, J., Wooltorton, J. R. A., Cohen, L. B., and Pieribone, V. A. (2012). Single action potentials and subthreshold electrical events imaged in neurons with a fluorescent protein voltage probe. Neuron $75,779-785$. doi: 10.1016/j.neuron.2012.06.040

Kamikouchi, A., Shimada, T., and Ito, K. (2006). Comprehensive classification of the auditory sensory projections in the brain of the fruit fly Drosophila melanogaster. J. Comp. Neurol. 499, 317-356. doi: 10.1002/cne.21075

$\mathrm{Li}, \mathrm{C}$., and Meinertzhagen, I. A. (1995). Conditions for the primary culture of eye imaginal discs from Drosophila melanogaster. J. Neurobiol. 28, 363-380. doi: $10.1002 /$ neu. 480280309

Lin, D. M., and Goodman, C. S. (1994). Ectopic and increased expression of Fasciclin II alters motoneuron growth cone guidance. Neuron 13, 507-523. doi: 10.1016/0896-6273(94)90022-1

Murthy, M., and Turner, G. (2010). "In vivo whole-cell recordings in the Drosophila brain," in Drosophila Neurobiology: A laboratory manual, eds B. Zhang, M. R. Freeman and S. Waddell (New York, NY:Cold Spring Harbor Laboratory Press), 534

Nesterov, A., Spalthoff, C., Kandasamy, R., Katana, R., Rankl, N. B., Andres, M., et al. (2015). TRP Channels in insect stretch receptors as insecticide targets. Neuron 86, 665-671. doi: 10.1016/j.neuron.2015.04.001

Pashley, D. H., Tay, F. R., Carvalho, R. M., Rueggeberg, F. A., Agee, K. A., Carrilho, M., et al. (2007). From dry bonding to water-wet bonding to ethanol-wet bonding. A review of the interactions between dentin matrix and solvated resins using a macromodel of th hybrid layer. Am. J. Dent. 20, 7-20.

Pereira, S. A., De Menezes, F. C., Rocha-Rodrigues, D. B., and Alves, J. B. (2009). Pulp reactions in human teeth capped with self-etching or total-etching adhesive systems. Quintessence Int. 40, 491-496.

Phan, N. T., Mohammadi, A. S., Dowlatshahi Pour, M., and Ewing, A. G. (2016). Laser desorption ionization mass spectrometry imaging of Drosophila brain using matrix sublimation versus modification with nanoparticles. Anal. Chem. 88, 1734-1741. doi: 10.1021/acs.analchem.5b03942

Rosay, P., Armstrong, J. D., Wang, Z., and Kaiser, K. (2001). Synchronized neural activity in the Drosophila memory centers and its modulation by amnesiac. Neuron 30, 759-770. doi: 10.1016/S0896-6273(01)00323-3
Schmitz, J., Hoger, U., Torkkeli, P. H., and French, A. S. (2012). Calcium buffering and clearance in spider mechanosensory neurons. J. Comp. Physiol. A. Neuroethol. Sens. Neural Behav. Physiol. 198, 477-483. doi: 10.1007/s00359012-0717-2

Seelig, J. D., Chiappe, M. E., Lott, G. K., Dutta, A., Osborne, J. E., Reiser, M. B., et al. (2010). Two-photon calcium imaging from head-fixed Drosophila during optomotor walking behavior. Nat. Methods 7, 535-540. doi: 10.1038/nmeth. 1468

Sharma, Y., Cheung, U., Larsen, E. W., and Eberl, D. F. (2002). PPTGAL, a convenient Gal4 P-element vector for testing expression of enhancer fragments in Drosophila. Genesis 34, 115-118. doi: 10.1002/gene.10127

Sinha, S., Liang, L., Ho, E. T., Urbanek, K. E., Luo, L., Baer, T. M., et al. (2013). High-speed laser microsurgery of alert fruit flies for fluorescence imaging of neural activity. Proc. Natl. Acad. Sci. U.S.A. 110, 18374-18379. doi: 10.1073/pnas.1216287110

Wilson, R. I., and Laurent, G. (2005). Role of GABAergic inhibition in shaping odor-evoked spatiotemporal patterns in the Drosophila antennal lobe. J. Neurosci. 25, 9069-9079. doi: 10.1523/JNEUROSCI.2070-0 5.2005

Wilson, R. I., Turner, G. C., and Laurent, G. (2004). Transformation of olfactory representations in the Drosophila antennal lobe. Science 303, 366-370. doi: 10.1126/science. 1090782

Wu, C. F., and Pak, W. L. (1975). Quantal basis of photoreceptor spectral sensitivity of Drosophila melanogaster. J. Gen. Physiol. 66, 149-168. doi: 10.1085/jgp.66.2.149

Conflict of Interest Statement: The authors declare that the research was conducted in the absence of any commercial or financial relationships that could be construed as a potential conflict of interest.

Copyright () 2016 Kay, Raccuglia, Scholte, Sivan-Loukianova, Barwacz, Armstrong, Guymon, Nitabach and Eberl. This is an open-access article distributed under the terms of the Creative Commons Attribution License (CC BY). The use, distribution or reproduction in other forums is permitted, provided the original author (s) or licensor are credited and that the original publication in this journal is cited, in accordance with accepted academic practice. No use, distribution or reproduction is permitted which does not comply with these terms. 


\section{APPENDIX}

\section{Goggatomy-Step by Step}

1. Clean blade with alcohol and snap into quarters.

2. Melt a small piece of soft dental wax onto a $12 \mathrm{~mm}$ circular coverslip, place in a $35 \mathrm{~mm}$ petri dish filled with DS (Figure A1A).

3. Cold anesthetize flies for $<30 \mathrm{~s}$.

4. Pick up a drop of LCR (volume just a little larger than the head) with a tungsten tip.

5. Lie fly on its side on the lid of the $35 \mathrm{~mm}$ petri dish. Cut off head with blade. Place head on its posterior surface.

6. Apply a droplet of LCR on the head to completely cover it (Figure A1B).
7. Place a drop of water on the LCR and apply the curing light for $1 \mathrm{~min}$, protecting eyes with goggles (Figure A1C).

8. Wipe off the saline with a tissue and apply a drop of DS.

9. Under higher magnification orient the fixed fly head. Hold the blade at an angle of $\sim 30^{\circ}$ to petri dish surface (Figure A1D). Cut through the embedded head just above the A2-A3 (Figure A1B) joint with a swift and firm motion.

10. Trim the cured LCR chip (Figure A1E) and transfer to the holding dish.

11. Insert the chip into the dental wax under saline, with the cut surface appropriately oriented (Figure A1F).

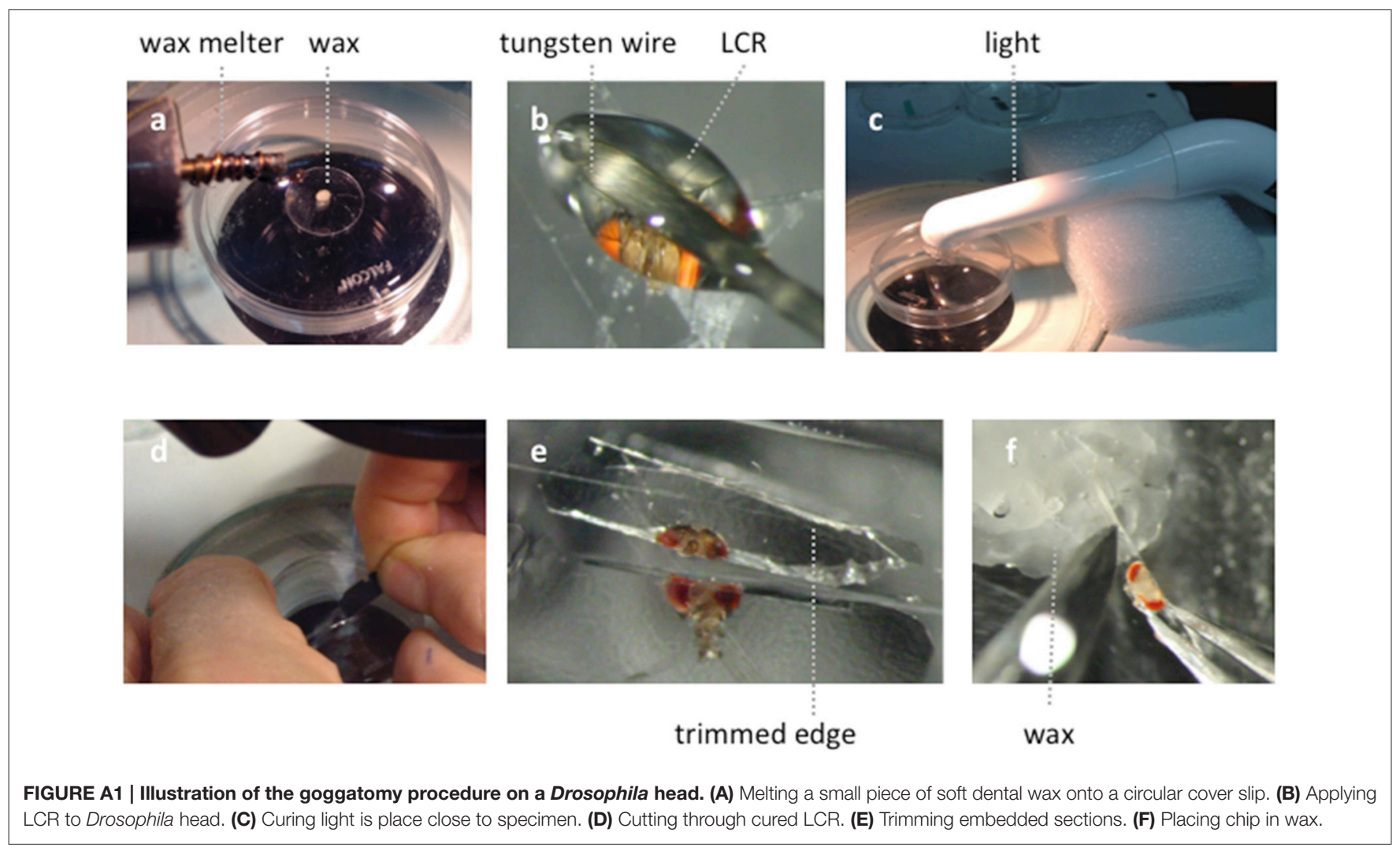

